\title{
COMMUTANTS CONTAINING A COMPACT OPERATOR
}

\author{
BY PAUL S. MUHLY 1
}

Communicated by Paul Halmos, October 9, 1968

All Hilbert spaces considered in this paper will be complex, and all operators will be bounded and linear. The algebra of all (bounded linear) operators on a Hilbert space $\mathfrak{H C}$ will be denoted by $\mathscr{L}(\mathfrak{H C})$. If $T$ is in $\mathscr{L}(\mathfrak{H C})$ and $\mathscr{T}$ is a subspace of $\mathfrak{F C}$ invariant under $T$ then the operator in $\mathscr{L}(\mathfrak{T})$ obtained by restricting $T$ to $\mathfrak{T} /$ will be denoted by $T \mid \Re$.

1. Introduction. Let $T$ in $\mathscr{L}(\mathfrak{F})$ be a contraction (i.e., $\|T\| \leqq 1$ ) and suppose that the minimal strong unitary dilation $U$ of $T$ is the bilateral shift of multiplicity one. In [5], Sarason proves two remarkable theorems for such operators. The first (Theorem 1) describes the commutant of $T$ in terms of that of $U$, while the second (Theorem 2) gives a necessary and sufficient condition that an operator in the commutant of $T$ be compact. In [4], Sz.-Nagy and Foias present a generalization of Sarason's first theorem to arbitrary contractions (cf. [2] for an elementary proof of the Sz.-Nagy-Foias theorem). Using the Sz.-Nagy-Foias result we are able to obtain a generalization of Sarason's second theorem. In this note we state this generalization and briefly outline the proof; complete details will appear elsewhere.

2. Statement of the theorem. Throughout, $\varepsilon$ will denote a fixed separable Hilbert space and $H_{\varepsilon}^{2}$ will denote the Hilbert space of all weakly measurable, norm square integrable $\varepsilon$-valued functions $f$ on the unit circle with the property that the negative Fourier coefficients of $f$ vanish. We will denote by $L_{\mathscr{L}(\varepsilon)}^{\infty}$ the space of all essentially bounded, weakly measurable $\mathscr{L}(\mathcal{E})$-valued functions on the unit circle. The space $H_{\mathcal{L}(\mathcal{E})}^{\infty}$ will be the subspace of $L_{\mathfrak{L}(\mathcal{E})}^{\infty}$ consisting of all functions whose negative Fourier coefficients vanish. For $A$ in $L_{\mathscr{L}(s)}^{\infty}$, $A^{*}\left(e^{i t}\right)$ is defined to be $\left(A\left(e^{i t}\right)\right)^{*}$.

Let $\Theta$ in $H_{\mathcal{L}(\varepsilon)}^{\infty}$ be inner, i.e., let $\Theta\left(e^{i t}\right)$ be unitary for almost all $t$, and let $\mathfrak{H C}=H_{\varepsilon}^{2} \ominus \Theta H_{\varepsilon}^{2}$. We consider operators $T$ which may be written in the form

$$
T=P U_{+} \mid \mathfrak{H C}
$$

1 These results are part of the author's doctoral dissertation written at the University of Michigan under the supervision of Professor R. G. Douglas. The research was partially supported by an NSF Graduate Fellowship. 
where $U_{+}$is the unilateral shift on $H_{\varepsilon}^{2}$ and $P$ is the projection of $H_{\varepsilon}^{2}$ onto $\mathfrak{H C}$. It is well known [3] that operators $T$ of the form (1) represent the most general contraction with the property that $T^{n}$ and $T^{*_{n}}$ converge strongly to zero. The Sz.-Nagy-Foias theorem says that an operator $S$ in $\mathcal{L}(\mathcal{F C})$ commutes with $T$ if and only if there exists $\Phi$ in $H_{\mathscr{L}(\varepsilon)}^{\infty}$ such that $\Phi\left(\Theta\left(H_{\varepsilon}^{2}\right)\right) \subset \Theta H_{\varepsilon}^{2}$ and $S=P \Phi \mid \mathcal{F}$.

Let $c^{\infty}(\mathcal{E})$ denote the Banach space of compact operators on $\varepsilon$ with the operator norm, and let $\boldsymbol{C}_{c^{\infty}(\varepsilon)}$ denote the space of continuous functions from the unit circle into $c^{\infty}(\mathcal{E})$.

With the preliminaries out of the way, we state our characterization of those operators $S$ in the commutant of $T$ which are compact.

THEOREM. Let $\mathfrak{K}=H_{\varepsilon}^{2} \ominus \Theta H_{\varepsilon}^{2}$ for some inner function $\Theta$ and let $T=P U_{+} \mid$FC where $U_{+}$is the unilateral shift on $H_{\varepsilon}^{2}$ and $P$ is the projection of $H_{\varepsilon}^{2}$ onto $\mathfrak{H C}$. Let $S$ commute with $T$ and suppose $S=P \Phi \mid \mathfrak{H C}$ where $\Phi$ is in $H_{\mathfrak{L}(\varepsilon)}^{\infty}$ and $\Phi\left(\Theta H_{\varepsilon}^{2}\right) \subset \Theta H_{\varepsilon}^{2}$. Then $S$ is compact if and only if

$$
\Theta^{*} \Phi \text { is in } H_{\mathcal{L}}^{\infty}(\varepsilon)+C_{c^{\infty}}(\varepsilon) \text {. }
$$

Notice that in case $\varepsilon$ is one-dimensional, our theorem is simply Sarason's second theorem.

3. Outline of the proof. The line of argument used in proving our theorem follows explicitly that of Sarason's proof. The complications which arise in our proof stem from the problem of finding and manipulating appropriate vector-valued analogues of certain classical results in $H^{\mathrm{p}}$ theory.

To prove the sufficiency, suppose $\Theta^{*} \Phi=\Omega+K$ where $\Omega$ is in $H_{\mathscr{Q}(\varepsilon)}^{\infty}$ and $K$ is in $C_{c^{\infty}(\varepsilon)}$; then $\Phi=\Theta(\Omega+K)$. It is possible to find an increasing sequence of finite rank projections $\left\{P_{k}\right\}$ on $\varepsilon$ and a sequence of Cesaro means $\left\{\sigma_{k}\right\}$ of the Fourier series of $K$ such that if $K_{k}\left(e^{i t}\right)$ $=P_{k} \sigma_{k}\left(e^{i t}\right) P_{k}$, then $\lim _{k \rightarrow \infty}\left\|K_{k}\left(e^{i t}\right)-K\left(e^{i t}\right)\right\|=0$ uniformly in $t$. If we set $\Phi_{k}=\Theta\left(\Omega+K_{k}\right)$, then it is clear that $P \Phi_{k} \mid \mathfrak{H C}$ converges to $P \Phi \mid \mathfrak{H C}$ in the norm on $\mathcal{L}(\mathcal{H C})$. Furthermore, a simple calculation shows that each $P \Phi_{k} \mid \mathcal{H C}$ is of finite rank. It follows that $P \Phi \mid \mathfrak{F C}$ is compact.

The proof of the converse is based on certain duality properties possessed by $H_{\mathcal{L}(\varepsilon)}^{\infty}+C_{c^{\infty}(\varepsilon)}$ which we now describe. Let $E$ be an arbitrary (complex) Banach space with dual $E^{*}$, and let $\boldsymbol{C}_{E}$ denote the space of continuous $E$-valued functions on the unit circle. It is well known [1], that $C_{E}$ is a Banach space and that its dual is $\mathrm{SBV}_{E^{*}}$, the space of all $E^{*}$-valued functions on the unit circle of strong bounded variation. Let $\mathfrak{A}_{E}$ denote the subspace of $\boldsymbol{C}_{E}$ consisting of all functions whose negative Fourier coefficients vanish, and let $H_{0 E^{*}}^{1}$ denote the space of Bochner integrable $E^{*}$-valued functions $f$ on the unit circle 
with the property that the nonpositive Fourier coefficients of $f$ vanish. The following generalization of the F. and M. Riesz theorem is valid.

Lemma. If $E$ is a Banach space with separable dual, then the anihilator of $\mathfrak{A}_{E}$ in $S B V_{E^{*}}$ may be identified with $H_{0 E^{*}}^{1}$.

The dual of $c^{\infty}(\varepsilon)$ is the space of trace class operators which we denote by $c^{1}(\mathcal{E}) ; c^{1}(\mathcal{E})$ is separable and its dual is $\mathcal{L}(\mathcal{E})$ (cf. [6]). If $L_{c^{1}(\varepsilon)}^{1}$ denotes the class of all Bochner integrable $c^{1}(\varepsilon)$-valued functions on the unit circle, then $L_{\mathcal{L}(\varepsilon)}^{\infty}$ is the dual of $L_{c^{1}(\varepsilon)}^{1}$. The lemma and these remarks show that the dual of $C_{c^{\infty}(\varepsilon)} / \mathfrak{A}_{c^{\infty}(\varepsilon)}$ is $H_{o c^{1}(\varepsilon)}^{1}$, and that the second dual of $C_{c^{\infty}(\varepsilon)} / \mathfrak{A}_{c^{\infty}(\varepsilon)}$ is $L_{\mathscr{L}(\varepsilon)}^{\infty} / H_{\mathscr{L}(\varepsilon)}^{\infty}$. The natural imbedding of $\boldsymbol{C}_{c^{\infty}(\varepsilon)} / \mathfrak{A}_{c^{\infty}(\varepsilon)}$ in $L_{\mathcal{L}(\varepsilon)}^{\infty} / H_{\mathcal{L}(\varepsilon)}^{\infty}$ carries the coset $K+\mathfrak{A}_{c^{\infty}(\varepsilon)}$ to the coset $K+H_{\mathcal{L}(\varepsilon)}^{\infty}$, where $K$ is in $C_{c^{\infty}(\varepsilon)}$. Thus to prove the necessity of (2), it suffices to prove that the linear functional $\lambda$ on $H_{o c^{1}(\varepsilon)}^{1}$ defined by

$$
\lambda(F)=\int \operatorname{tr}\left(\Theta^{*} \Phi F\right) d m, \quad F \text { in } H_{o c^{1}(\varepsilon)}^{1}
$$

(where $m$ is normalized Lebesgue measure) is continuous with respect to the weak-star topology on $H_{o c^{1}(\xi)}^{1}$. The proof of this is achieved by making use of a series of delicate approximations to reduce the problem to the case when $\varepsilon$ is finite dimensional. Sarason's arguments then apply mutatis mutandis to give the result. We remark that the approximation arguments rely heavily on the properties of compact operators, and that a fundamental role is played by a generalization of a factorization lemma of F. Riesz due to Sarason [5, Theorem 4].

4. Concluding remarks. Our theorem enables us to prove the following

CoROllaRy. Let $T$ be an operator written in the form (1) for some inner function $\Theta$. Then $T$ is compact if and only if $\Theta$ can be written $\Theta\left(e^{i t}\right)=e^{i t}+K\left(e^{i t}\right)$ where $K$ is a $c^{\infty}(\mathcal{E})$-valued function on the unit circle which has an analytic extension to some neighborhood of the closed unit disk.

On the basis of our results we are able to find invariant subspaces for operators belonging to a class which contains the compact operators.

ADDED IN PROOF. It has been brought to our attention that Ryan (Indag. Math. 25 (1963), 408-412) has proven a theorem more general than our lemma by methods different from ours. 


\section{BiBLIOGRAPHY}

1. N. Dinculeanu, Vector measures, Pergamon Press, New York, 1967.

2. R. G. Douglas, P. S. Muhly and C. Pearcy, Lifting commuting operators, Michigan Math. J. 15 (1968), 385-395.

3. B. Sz.-Nagy and C. Foiaş, Analyse Harmonique des Opérateurs de l'Éspace de Hilbert, Akad. Kiad6, Budapest, 1967.

4. - Dilatations des commutants d'opérateurs, C. R. Acad. Sci. Paris 266 (1968), 493-495.

5. D. E. Sarason, Generalized interpolation in $H^{\infty}$, Trans. Amer. Math. Soc. 127 (1967), 179-203.

6. R. Schatten, Norm ideals of completely continuous operators, Springer-Verlag, Berlin, 1960.

University of Michigan, Ann Arbor, Michigan 48104 